\title{
A Macroscopic Inception Criterion for the Upward Leaders of Natural Lightning
}

\author{
Udaya Kumar, Prasanth Kumar Bokka, and Jagannath Padhi
}

\begin{abstract}
The increased use of electronic equipments in day-to-day life as well as the steady increase in the height of the structures have considerably increased the lightning threat. As a result, a suitable lightning protection system has become very essential. For a reliable evaluation of the protection efficacy the process involved with the final bridging needs to be accurately ascertained. This requires tracing of the inception as well as the propagation of the upward connecting leaders from both the protection system as well as the structure being protected. Owing to the complexity of the problem, only the macroscopic models seem to be practical. As a first step in this direction, the present work has developed a model capable of ascertaining the inception of the upward leaders from both the air termination network as well as the structure itself. In this, the discharge activities around the prospective inception regions are represented by a corona envelope, which is followed by a streamer. Using the works based on the long air-gap switching surge breakdown experiments it is shown that the streamer in the model must attain a critical length for the inception of a leader. This critical length is shown to be sensibly independent of gap-geometry and the electrode radii when varied below the critical radii. The applicability and the stability of the model is demonstrated with a few salient examples.
\end{abstract}

Index Terms-Connecting leaders, leader inception, lightning protection, upward discharges.

\section{INTRODUCTION}

$\mathbf{T}$ HE increased use of sensitive electronic equipment along with the steady increase in the height of commercial and industrial structures have considerably increased the threat due to natural lightning. Due to which, a reliable protection of ground based structures/systems against natural lightning has become imperative. Lightning "prevention" or "protection" in an absolute sense is rather impractical. However, the detrimental consequences can be drastically reduced by employing suitable Lightning Protection System (LPS).

The air termination network of the LPS is mainly responsible for the stroke interception - the first and foremost task in a protective action. For shorter structures and for the cases wherein the increase in net field is not sufficient, then the stroke interception would be mostly through upward streamers. On the contrary, for tall structures and cases where there is a sufficient net field well in advance of bridging, the associated process can be split into two important stages: (i) inception of upward

Manuscript received April 7, 2004; revised June 23, 2004. Paper no. TPWRD00160-2004.

U. Kumar and P. K. Bokka are with the Department of High Voltage Engineering, Indian Institute of Science, Bangalore, India, 560012 (e-mail: uday@ hve.iisc.ernet.in; prasant@hve.iisc.ernet.in).

J. Padhi is with the Bharath Heavy Electricals Limited, India (e-mail: jpadhi@rediffmail.com).

Digital Object Identifier 10.1109/TPWRD.2005.844273 connecting leaders, and (ii) their extension/propagation toward the descending leader. For a reliable assessment of the lightning attachment and hence the protection efficacy, both the above needs to be attended. The bridging by streamer mechanism is relatively simple to ascertain and hence additional efforts are not called for. It is just sufficient to check for the existence of critical average gradient between the descending leader tip and the nearest point on the grounded object. However, same thing is not true with the case, where significant upward leader activities are present. Further, the field observations clearly indicate that the edges and corners of tall structures/buildings can be quite active in launching upward discharges. Therefore, apart from considering the upward connecting leaders from the air termination network, it becomes equally important to consider the same from the structures to be protected. Once a connecting leader is incepted, its propagation characteristics can be expected to be less dependent on the geometry of the launching region. In summary, the first and foremost task in the evaluation of the lightning attachment to tall structures and for the cases where significant upward leader activities can be expected is to consider the inception of upward leaders. The present work basically attempts to address the same.

\section{LITERATURE SURVEY}

Power transmission lines were extended over large geographical areas and as a consequence were subjected to a significant amount of lightning threat. This has lead to many investigations and significant progress on the modeling of the final stages of bridging or the attachment process.

The well-known Electro Geometric Model (EGM), which is based on the principle of striking distance, was one of the important developments. The striking distance is defined as the "distance between the object to be struck and the tip of the downward-moving leader at the instant that upward connecting leader/streamer is initiated from the object" [1]. In other words, attempt is made to consider both the stages of the bridging process simultaneously. This striking distance is governed by the voltage of the descending leader tip, which in turn is dependent on the charge on the leader. By using the field data, the charge on the leader has been related to a more familiar entity, return stroke current peak $I_{p}: I_{p}=10.6 Q^{0.7}$, where $Q$, is the total charge on the leader. Armstrong and Whitehead [2] have developed an analytical expression for striking distance $r_{s}$ (m) in terms of the amplitude of the prospective return stroke current $I_{p}(\mathrm{kA}): r_{s}=a I_{p}^{c}$. Where, $a \& c$ are constants.

Many other researchers have also arrived at the same equation but with different values for $a$ and $c$. The lightning task force of 
IEEE [3] has compiled them and the range of values for $a$ is reported to be between $6-10$, and that for $c$ it is between $0.6-0.8$. The final bridging is obviously dependent on the net field distribution and hence can be expected to show some dependency on the geometry of the object. For this, suitable gap factors were arrived using the results of laboratory experiments and the field observation data [4]. This gap factor is reported to vary between 1-1.2 for towers, conductors and the ground. These gap factors are not general so as to be extended to general 3D structures and also even to towers and conductors of height greater than that encountered with the transmission lines.

The Rolling Sphere Method [1] is one of the direct applications of the EGM for 3D geometries. It is relatively simple to use and hence commonly employed for the analysis and design of LPS. However, it has the following serious limitations:

- The expression for the striking distance does not show any explicit reference to the field intensification caused by the structure geometry.

- The inception and propagation of the upward leaders are not considered rigorously. The final bridging envisaged appears to be closer to a streamer mechanism rather than leader-streamer based process.

Eriksson [5], Dellera et al. [6] and Rizk [7], [8] have considered modeling of the attachment to towers and conductors. Invariably, they have employed the critical radii concept to determine the ambient field necessary for the upward leader inception. Rizk, using his earlier work on switching surge breakdown of long air gaps [9], has made a more detailed account of the leader characteristics and has arrived at a height dependent critical radii. As the above three models dealt with isolated towers and conductors, they cannot to be applied to general structures. This deficiency arises because of the difference in the field distribution at the respective structure tops.

In a recent work [10], the above limitations were identified and modifications were adopted in analysing a closely distributed lightning protection system involving towers interconnected by ground wires. Again, this method is not general and hence not applicable to structures.

For deciding the conditions necessary for the inception of upward leaders, Kumar \& Gosh [11] and Alesandrov [12] have attempted to directly apply the concept of Critical Radii (CR) to masts/catenoids on the buildings. Clearly, the inception of the upward leaders is governed by the field distribution in and around the inception regions and this distribution is very significantly different for masts/catenoids placed over the buildings as compared to isolated masts/conductors. Further more, even for sphere-plane and conductor-plane geometries the critical radii is not independent of the gap [9], [13]. More importantly, the above approach is incapable of predicting the inception of upward leaders from building itself.

Alternatively, the attainment of the critical field $(30 \mathrm{kV} / \mathrm{cm})$ over an effective space charge corona radius of $\sim 0.3 \mathrm{~m}$ has been employed for determining the inception of upward leaders [14]. This method has not been validated with the results of long air gap switching surge breakdown experiments. Also, as the critical radius for conductor-plane geometry is about $10 \mathrm{~cm}$, this approach seems to be not applicable. In other words this approach is not general and reliable.

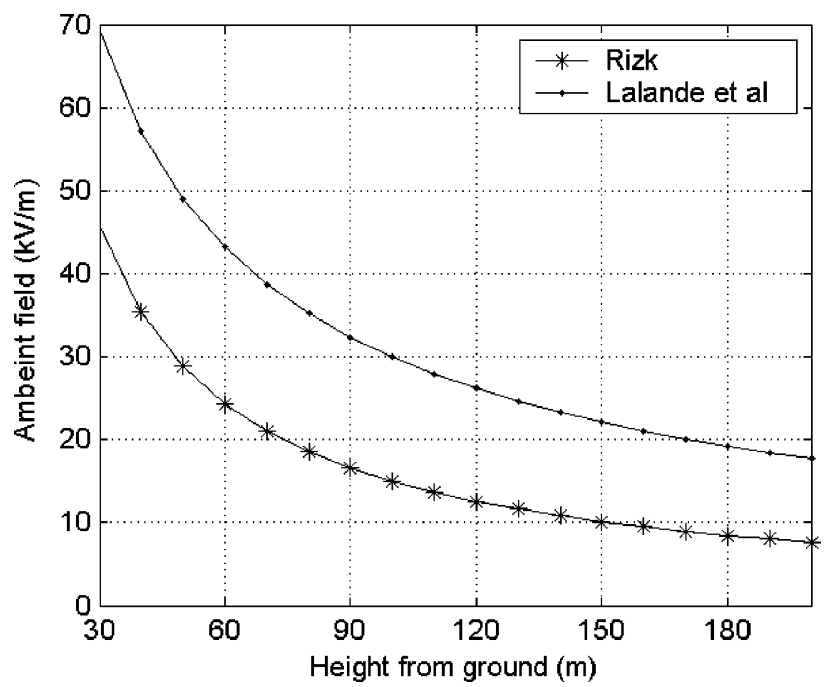

Fig. 1. Ambient field at the inception of continuous leader.

The other concept employed for determining the instant of upward leader inception is the Critical Range of Field Intensification (CRFI) [12]. It is based on some of the findings of the long-air gap switching surge breakdown experiments. However, the CRFI concept seems have not been tested with well-established long-air gap switching surge breakdown results. Also, it is not clear whether it can be applied to conductor-plane like geometries, building edges etc. As the field intensification sought in the method rely on the field in and around $1 \mathrm{~m}$ or so form the inception point, it becomes necessary to differentiate between the various geometries. Also, when the radius of the electrode is increased toward the critical radii, a stage will be reached at which the above criterion by itself does not guarantee even the critical field at the electrode surface.

There is also a model for the upward discharge phenomena [15] from vertical conductor (mast), which is based on the governing physical processes. It is quite complicated and extremely difficult to apply to lightning environment. In view of this, some simplified version has also been put forth in the same work [15]. However, it is not clear whether the simplification is extendable to arbitrary geometry. More investigations in this direction would be a very useful. Furthermore, the Rizk work [7]-[9], which has shown very good matching with the experimental results on breakdown, length of final jump etc., suggests as shown in Fig. 1, lower values of ambient field for the inception of the upward leaders. The corresponding ambient potential predicted by Rizk work saturates around 1.5 MV, whereas that due to Lalande et al. [15] exhibits a continuous increase. As the height increases, the field pattern around the tip of the mast/conductor extending up to a few meters can be expected to become almost independent of the height. In addition, the ambient field becomes a very small fraction of the total field. Therefore, the height dependency of the ambient potential, required for the continuous leader inception from masts and conductors, should tend to saturate. In view of this, the present work basically relies on the Rizk's work. It may be opined here that once a stable leader is established, the influence of the electrode on the net field ahead of the leader progressively diminishes. 
In summary, for a reliable evaluation of the lightning protection to tall structures, it is very essential to appropriately consider the inception and propagation of the upward leaders from both protection system, as well structure being protected. This demands a suitable general model for the inception of upward leaders and the pertinent survey of literatures indicates that the available models possess some serious limitations. In view of this, the present work aims to develop a simple macroscopic criterion for the inception of the positive upward leaders from any arbitrary geometry.

Similar to most of the earlier works, the possible roles of space charges distributed in the space between the cloud and the ground/grounded object and the time rate of rise of ambient field on the inception of upward leaders are not considered presently. In most of the geographical locations, the negative lightning dominates by more than $90 \%$ and due to this, most of the earlier works have dealt only with negative lightning. The present work also limits itself to the negative cloud-to-ground lightning. Further, upward flashes are not considered, as they do not come under the regime of classical lightning protection schemes.

\section{PRESENT Work}

It would be very ideal if all the microscopic phenomena leading to the inception of the upward leader could be modeled. However, at present it appears to be almost a formidable task. Considering the complexity of the phenomena as well as the amount of computational burden, only the macroscopic models seem to be practical.

In the lightning environment, the gap between the descending leader and the inception point, at the time of upward leader inception will be considerably larger than the typical laboratory gaps. This will be especially true for tall structures. As a first approximation, this gap can be estimated to be ranging above 20-50 m. At such gaps, the field pattern around the inception point will be little influenced by the spatial extent of the source. In other words, the activities in and around the inception region will become independent of the source geometry.

In this regard, it was envisaged that the streamer extension at the macroscopic level should attain a certain minimum level for the conversion into a stable or the continuous leader. If this were to be true then the equivalent streamer extension at the inception of a continuous leader must approximately be the same for both long sphere-plane and conductor-plane geometries. The commonly discussed rod-plane gap possesses a field variation, which is slower than that with sphere-plane gap. It may be noted here that the field patterns prevailing in the above two geometries encompass a wide range of practical situations. A good support for the above proposition concerning the critical streamer lengths, even though gaps studied there are much smaller, is available from the work of Brambilla et al. [13, Discussion in 9 by Pigini]. By using the work of Gallimberti, they have shown that for electrode of critical radii and gaps ranging up to $8 \mathrm{~m}$, the computed streamer lengths at the leader inception for both sphere-plane and conductor-plane geometries remains the same.

A stronger verification of the present proposition would be possible with the experimental results on long air gaps, stressed

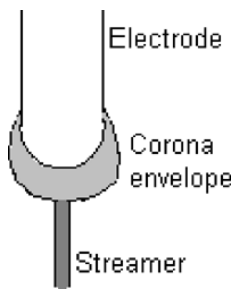

Fig. 2. Schematic of the proposed model.

with critical switching impulses or with their extension. This intended verification requires a suitable modeling of the streamer extension. Various simplified criteria have been proposed in the literature. Important ones are: (i) based on the extension of the geometric field for specified gradient $\mathrm{E}_{\mathrm{st}}$ \& (ii) extension of the streamer modeled as a uniform cylindrical charge with an average gradient of $\mathrm{E}_{\mathrm{st}}$. As the performance of the above two criteria was found to be not satisfactory, the present work proposes alternative criterion. In this the streamer is modeled as a cylindrical charge distribution with an internal gradient of $\mathrm{E}_{\mathrm{st}}$. The radius of the streamer, which is assumed to be uniform throughout, is fixed iteratively such that the average surface gradient is $\mathrm{E}_{\mathrm{c}}=24 \mathrm{kV} / \mathrm{cm}$. This model is further augmented by a modification in which, whenever is essential, a corona envelope surrounds the critical region (refer Fig. 2). Details will be given later. The envelope of the corona sheath is dictated by a surface gradient of $\mathrm{E}_{\mathrm{c}}$ and inside gradient of $\mathrm{E}_{\mathrm{st}}$.

Now with regard to the gradient inside the streamer $\mathrm{E}_{\mathrm{st}}$, it is well established that its value ranges from $4-5 \mathrm{kV} / \mathrm{cm} \mathrm{[16].}$ Many of the recent works [7], [10] have taken this value to be $4 \mathrm{kV} / \mathrm{cm}$ and the present work uses the same. In the numerical simulation, the streamer is divided into small segments and a linear charge variation is assumed within each segment. The unknown charge magnitudes are determined then by enforcing an internal gradient $\mathrm{E}_{\mathrm{st}}$. The streamer radius, which is made uniform throughout and its length are extended so as to have $E_{c}$ at its surface and the tip. The surface gradient is met only in the average sense. An attempt to model radius varying according to the charge distribution along the length was found to initiate numerical difficulties. Invariably, the extension of this equivalent streamer would be lesser than the extension of the geometric field determined by $\mathrm{E}_{\mathrm{st}}$. It may be noted here that the model proposed is not limited by the values assumed for various gradients.

\section{A. Numerical Method for the Field Computation}

An accurate computation of the associated electrostatic field is very essential. The problem is basically of open geometry type and requires good accuracy at the sharp edges/corners. Considering these, the charge simulation method is selected for the field computation. However, it may be noted here that the model proposed in this work is not limited by the method employed for the field computation and hence any method could be utilized. The structure geometry along with the mast and catenoids were discretised mostly by point charges. The selection of point charges yields two advantages: firstly, the nonuniform Discretization, which is very essential to control the computational burden, can be made relatively easier, and secondly, it allows the use of pointers for array manipulation in MATLAB (which is used as the computational platform). 
The use of MATLAB significantly simplifies the code development as well as the subsequent simulation work. The definition of variables and functions (or subroutines) are far more simplified than that with general-purpose higher-level languages. For example there is no need to declare the variables and functions apriori. Further, versatile debugging facility makes it easier to trace the program run and hence the errors. The large class of built in mathematical functions prove very handy. For example very robust and efficient matrix solution and condition checking routines are available. The extensive $2 \mathrm{D}$ and $3 \mathrm{D}$ plotting facilities makes the graphical representation of the results very simple. They aid in the visualization and interpretation of the simulation results. In addition to all these, the codes written in MATLAB are machine and operating system independent and hence can be easily ported across various platforms.

The simulation requires that at each and every instant the ambient potential due to descending leader must be evaluated on the surface of the structure and that on the mast/catenoids. Furthermore, once the upward streamer is initiated, the potential distribution inside the streamer and the electrostatic interaction of the streamer with the structure (including the air termination network), must all be properly considered. The necessary equations for implementing these can be found in an earlier publication [10].

For the verification of the proposition regarding the critical length of the equivalent streamer at the inception of a continuous leader, simulations are carried out for long air-gaps first.

\section{B. Simulation Results for Sphere-Plane and Conductor-Plane Geometries}

Rizk [9] has validated his theory with the experimental results for sphere-plane gap of up to $25 \mathrm{~m}$ and conductor-plane gap of up to $15 \mathrm{~m}$. In view of this, his expressions will be the basis for the intended scrutinization. Further, as the actual gaps involved with lightning are much higher than the above gap spacing, it will be necessary to employ much larger gaps for the determination of the critical length for the equivalent streamer.

For the large gaps say greater than $20-50 \mathrm{~m}$, the field pattern up to a radius of say $3 \mathrm{~m}$ from the electrodes is practically unaffected by the presence of the plane. In other words the field pattern becomes almost independent of the gap. As a consequence, for a given electrode geometry, the voltage required for the inception of the continuous leader should be saturating. The same reasoning also demands that the critical radii should also exhibit saturation. It is mentioned in the literature that [6] the critical radii for conductor-plane geometry is expected to saturate at $10 \mathrm{~cm}$. For the sphere-plane geometry, the Rizk's work [9] shows that the curves for both the inception voltage as well as the critical radii show a saturating trend.

After making a detailed study on the Rizk's equations for both the continuous leader inception voltage as well as that for the critical radii, a gap of $50 \mathrm{~m}$ was selected first for the analysis. At this gap, the extension of the equivalent streamer should be the saturated value for conductor-plane geometry and close to saturation for the sphere-plane geometry (according to the Rizk's equation the percentage increase in leader inception voltage between $50 \mathrm{~m}$ to $500 \mathrm{~m}$ gap is about $2 \%$ ). The value of the critical radii for the above sphere-plane geometry is about $48 \mathrm{~cm}$.

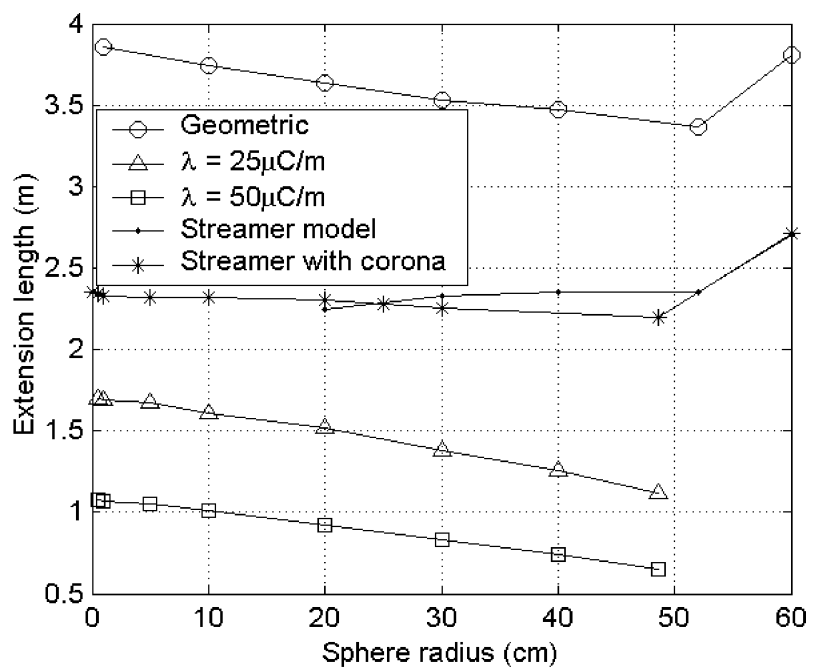

(a)

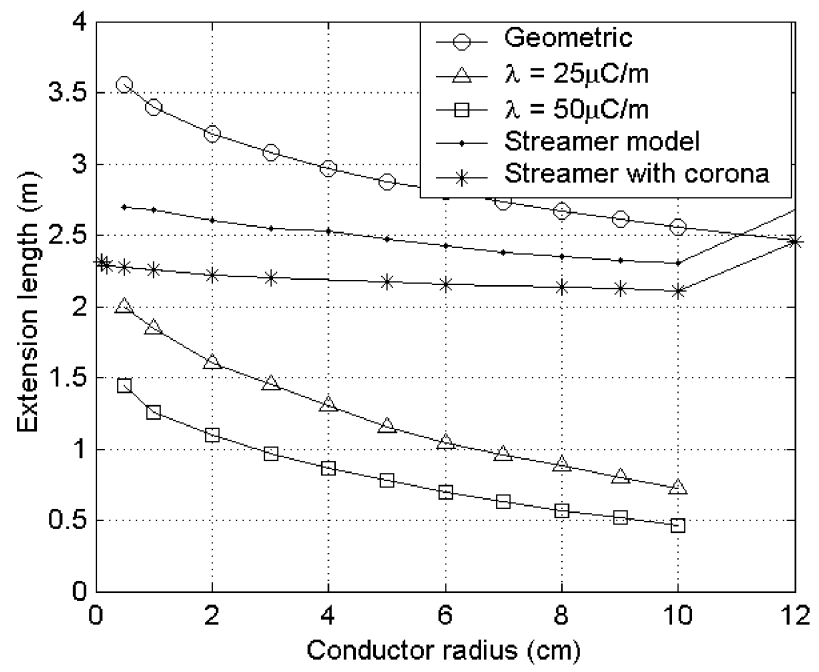

(b)

Fig. 3. Comparison of the critical streamer lengths as predicted by the four macroscopic models. (a) Sphere-plane geometry. (b) Conductor-plane geometry.

Using the Rizk's equations, the respective continuous leader inception voltages are applied to either geometry (with different values of electrode radii) and the equivalent streamer lengths are determined. This continuous leader inception voltage remains constant till the electrode radius approaches the critical radii (for that gap) and increases thereafter. In the following, the streamer length given by the four of the models discussed will be presented.

1) Geometric Field Extension: According to this the streamer is assumed to extend up to a point satisfying the average potential gradient $\mathrm{E}_{\mathrm{st}}$, Where $\mathrm{E}_{\mathrm{st}}$ is the stable streamer gradient $(3-5 \mathrm{kV} / \mathrm{cm})$. In this, the streamer is not modeled and hence the computation is very simple.

Fig. 3(a) and (b) shows the streamer extensions with $\mathrm{E}_{\mathrm{st}}=$ $4 \mathrm{kV} / \mathrm{cm}$ for both sphere-plane and the conductor-plane geometries. It can be seen that the variation of streamer length is greater than $35 \%$ for conductor-plane geometry and greater than $10 \%$ for sphere-plane geometry. In addition, the average values of the extension length for either geometry differ by $24 \%$. 
TABLE I

SumMary OF THE STREAMER LENGTHS FOR THE FOUR MODELS

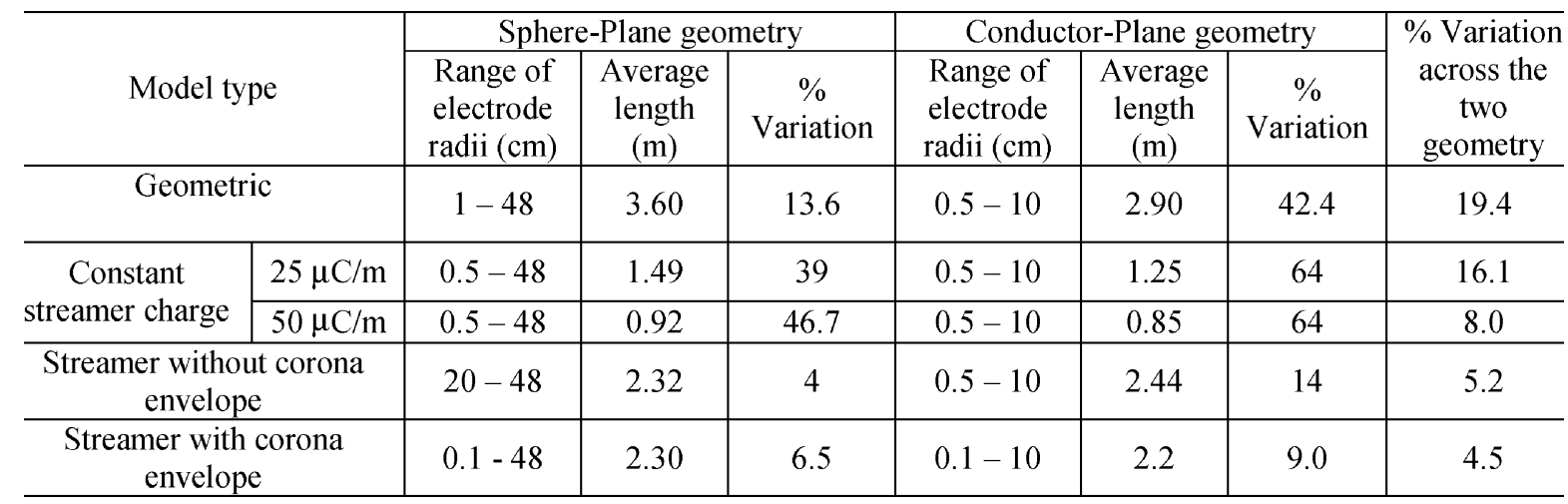

2) Uniform Cylindrical Charge Distribution: In this, whole of the streamer is modeled as cylindrical section of constant charge density, whose value being taken from results of long air gap breakdown experiments. The streamer is extended to a point such that average gradient (net field) within the streamer is equal to $\mathrm{E}_{\mathrm{st}}(4 \mathrm{kV} / \mathrm{cm})$. The radius of the streamer is determined by setting the surface gradient to $\mathrm{E}_{\mathrm{c}}$.

Fig. 3(a) and (b) shows the simulation results obtained for this model. The streamer extension exhibits larger variation $40 \%$ and $64 \%$ for sphere-plane and conductor-plane geometries, respectively. In addition, the average values of the extension length for either geometry differ by $64 \%$.

3) Piece-Wise Linear Charge With Uniform Axial Gradient and Radius: This is the first model proposed in the present work. Here streamer is modeled as cylindrical sections of linear charge distribution with an axial gradient of $\mathrm{E}_{\mathrm{st}}$ [17]. The streamer radius and length are iteratively varied so as to reach $E_{c}$. As mentioned earlier, whole of the model streamer possesses a single uniform radius corresponding to the critical surface field $\mathrm{E}_{\mathrm{c}}$ determined by the average linear charge density. Fig. 3(a) and (b) gives the results obtained for this model.

Due to the stability problems encountered with the sphere-plane geometry, the streamer extensions were computed only for electrode radii greater than $20 \mathrm{~cm}$. For the conductor-plane geometry, the range of electrode radius was $0.5-10 \mathrm{~cm}$. The matching of the streamer extension, at least for the range of electrode radius studied, seems to be good across the two geometries. However, the variation within the conductor-plane geometry was about $14 \%$. Further, when applied to lightning environment, the ambient potential at the leader inception, as predicted by this model, exhibits some appreciable dependency on the mast radii, even when the value of the radius is below the critical value.

4) The Above Streamer Model With a Corona Envelope Covering the Critical Zones: In the previous model, the over dependency of the streamer length on the electrode radii was due to the total neglecting of the discharge activities surrounding the electrode. In order to overcome this, of course with an additional computational burden, electrodes are covered with a corona envelope. This can be considered as an attempt to macroscopically represent the discharge model presented in [18]. It may be worth mentioning here that this envelope becomes essential only for electrodes whose radius is less than the critical radii.
The shape of the envelope is determined by the conditions that the gradient inside is $\mathrm{E}_{\mathrm{st}}$ and that on the boundary it is $\mathrm{E}_{\mathrm{c}}$. The streamer is modeled in the same way as the previous model and it is made to start from the corona envelope. The formation of the corona envelope has significantly improved the field distribution. For this model, the radius of the simulated streamer was reasonably consistent. For the sphere-plane geometry it varied from $4-4.5 \mathrm{~cm}$ and the same for the conductor-plane geometry was between $2.64-2.76 \mathrm{~cm}$. For streamer without corona, these values were $6-11 \mathrm{~cm}$ and $3.1-7.6 \mathrm{~cm}$ respectively.

As can be seen from Fig. 3(a) and (b), in this method the computed net streamer lengths are consistent across wide range of electrode radii. The net streamer length is the total length of the streamer including the thickness of the corona envelope at its root. The stability problems encountered with the previous model are eliminated. Further, the streamer lengths across the two geometries exhibit an appreciable matching. This model seems have put forth a first order field based approximation to the physical conditions prevailing in the active regions surrounding the electrodes.

The overall quantitative comparisons for the above four approaches are presented in Table I. It is very evident from the table that the streamer lengths predicted by the last model (i.e., second model proposed in the present work) exhibit reasonable invariance over a wide range of electrode radii and across two extreme cases of the field distribution. Therefore, it serves as a strong proof for the proposition made in this work that the equivalent streamer should attain a critical length for the inception of a continuous leader. In view of this, the above model can be considered as a very general model for the evaluation of the inception of positive upward leaders during natural lightning. It may be observed from the same figures that for electrodes with radii greater than the critical radii, the computed streamer length exceeds the above critical value. Further progressing on the same lines, it was decided to re-ascertain the value of critical streamer lengths for gaps of $20 \mathrm{~m}$ and $200 \mathrm{~m}$. These are probably close to the two extremities of the range of striking distances associated with the natural lightning. The results obtained for these gaps are in line with the previous deductions and the critical streamer lengths obtained are $2.1 \mathrm{~m}$ and $2.3 \mathrm{~m}$ respectively. These values are within $5 \%$ of the value obtained for $50 \mathrm{~m}$. In view of this, the critical streamer length necessary for the inception of a continuous leader is taken as $2.2 \mathrm{~m}$. 
The basic steps involved in the proposed work can be summarized as follows:

1) Descend the main leader till the net field in the critical zones attains the critical field $\mathrm{E}_{\mathrm{c}}$. The net field is the vectorial sum of the ambient field and the field produced by the induced charges on the structure as well as the ground. The induced charges on the structures are computed by field computation, wherein, the ambient potential produced by the descending leader serves as the source.

2) By forcing $E_{s t}$ along the maximum field line, simulate the equivalent streamer and check whether its length reaches the critical length of $2.2 \mathrm{~m}$. If so upward leader has incepted and hence stop, Else

3) Descend the main leader further down, first stabilize the corona envelope and then find the streamer extension. Repeat this till streamer reaches the critical length of $2.2 \mathrm{~m}$ (this length includes the thickness of the corona envelope at the streamer root).

\section{Verification With Tall Towers and Conductors}

For a further scrutiny, the above findings will be now applied to the lightning environment. Rizk [7], [8] works on lightning attachment to towers and conductors demands that the ambient potential at the leader inception must be nearly insensitive to the height and it must be independent of the radii of the masts/conductors when varied below the respective critical radii. For the intended verifications, it was not necessary to vary the air density, temperature and humidity and hence they are assumed to be constant. Their effect is basically reflected in the corresponding critical gradients.

Table II and Fig. 4 gives the simulation results. It can be observed that the ambient potential required for the inception of the upward leaders is reasonably insensitive to the radii of the mast/conductors. However, as the height of the mast/conductor is increased, some deviation from the Rizk's work could be seen. This in our opinion can be basically attributed to the following. The Rizk's equations for the ambient potential necessary for the leader inceptions were basically extended from his work on the analysis of breakdown in long air gaps stressed with switching surges. However, in contrast to long air gap experimentation, during lightning, the whole of the mast/conductor do not experience the same level of ambient potential/field.

As a result, the field distribution around the mast/conductor will now be of slightly lower strength as compared to that produced in long air gap configuration. This would in turn demand a small increase in the ambient potential required for the leader inception. With this justification, it can be concluded that the model proposed in the present work performs equally well even for the lightning environment.

For proving the numerical stability of the proposed model, it was applied to mast of $1 \mathrm{~mm}$ radius and a conductor of $2 \mathrm{~mm}$ radius. The computed ambient potentials at the inception for a $50 \mathrm{~m}$ height were within respectively, $7 \%$ and $14 \%$ of the value required by the Rizk's equations.

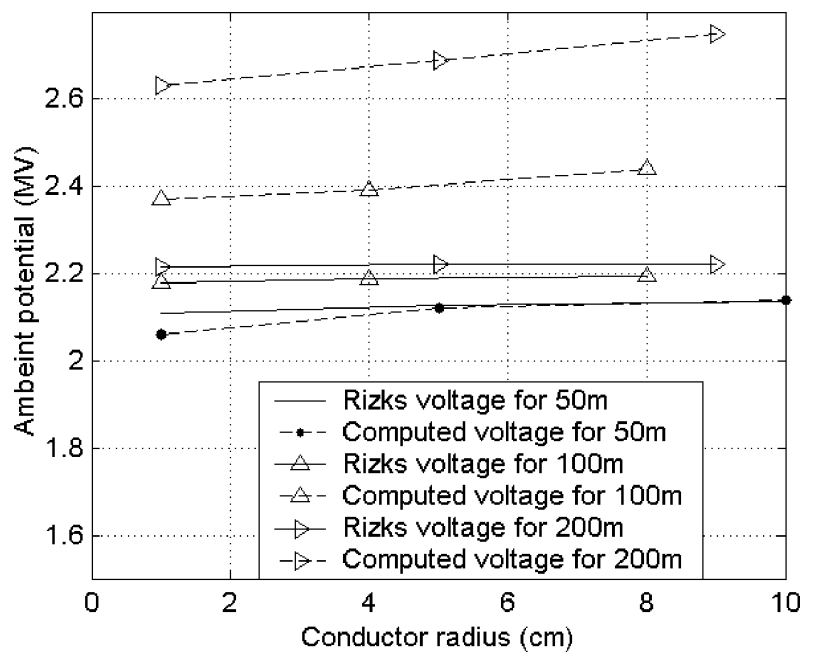

Fig. 4. Ambient potential at leader inception for different conductor height and radii.

TABLE II

AMBIENT POTENTIAL REQUIRED FOR LEADER INCEPTION FROM ISOLATED MASTS OF DIFFERENT HEIGHT AND RADIUS

\begin{tabular}{c|c|c|c|c|c}
\hline \multirow{2}{*}{$\begin{array}{c}\text { Mast } \\
\text { Length }\end{array}$} & \multirow{2}{*}{$\begin{array}{c}\text { Rizk } \\
\text { voltage }\end{array}$} & \multicolumn{4}{|c}{$\begin{array}{c}\text { Computed Leader Inception } \\
\text { Voltage (MV) }\end{array}$} \\
\cline { 3 - 6 } & & \multicolumn{4}{|c}{ Mast Radius (cm) } \\
\cline { 3 - 6 } & & 1 & 10 & 25 & 40 \\
\hline 25 & 1.346 & 1.25 & 1.27 & 1.28 & 1.3 \\
\hline 50 & 1.443 & 1.40 & 1.42 & 1.43 & 1.47 \\
\hline 75 & 1.479 & 1.44 & 1.45 & 1.50 & 1.54 \\
\hline 100 & 1.497 & 1.48 & 1.53 & 1.56 & 1.59 \\
\hline 150 & 1.516 & 1.52 & 1.60 & 1.61 & 1.68 \\
\hline 200 & 1.526 & 1.54 & 1.61 & 1.64 & 1.73 \\
\hline
\end{tabular}

\section{Application to Practical Structures}

For the purpose of illustrations, the proposed model will now be applied to practical cases. First, upward leader inception from a mast on a cylindrical building will be considered. The available methods, as has been discussed earlier have serious limitations making them unsuitable for the task. The commonly employed striking distance approach do not attend to the field intensification caused by the geometry and hence treats both the building top as well as masts almost identically. The established works on isolated masts/tower dealt only with that geometry. The field strength around the mast on a building will be significantly different from that on an isolated mast and therefore, methods developed for isolated mast/tower cannot be extended to mast on the building. At the same time, as discussed earlier, the recent propositions like CR, CRFI etc. have not been verified either with available results on long air gap breakdown experiments or with their extensions. Therefore, they cannot be reliably employed. It is worth noting here that the example under consideration is a typical commonly encountered lightning protection problem. Under such practical situations, the model proposed in the present work forms a very valuable tool. 


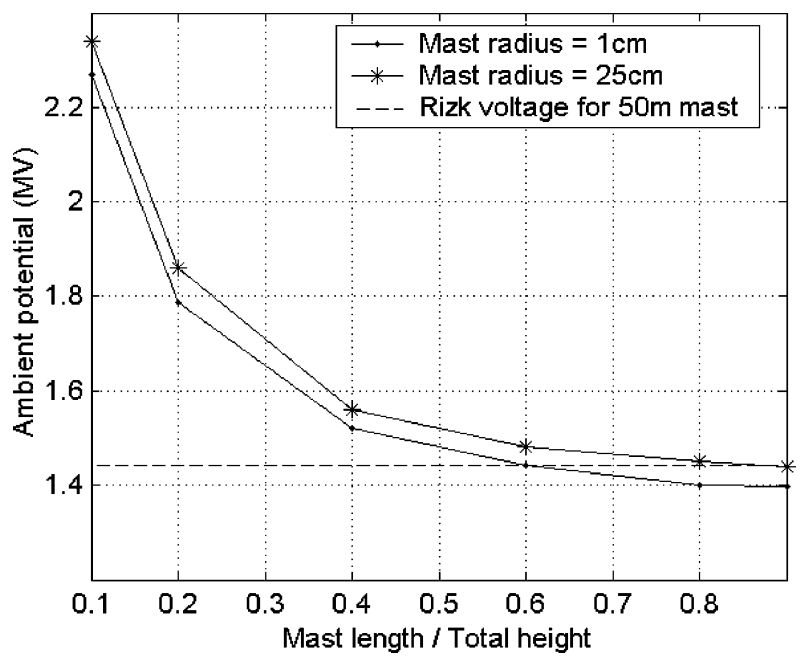

Fig. 5. Ambient potential at inception for a mast on the top of cylindrical building of radius $10 \mathrm{~m}$ (Total height $=50 \mathrm{~m}$ ).

Simulations are carried out for the above case by varying both the mast radius as well as the mast length. However, the effective height of the mast tip is held at $50 \mathrm{~m}$. In other words, building height is reduced for longer masts so as to keep the total height the same. Two values of radii $1 \mathrm{~cm}$ and $25 \mathrm{~cm}$ are considered for the mast and the masthead is made hemispherical. The computed ambient potentials required for the inception of the upward leaders are plotted in Fig. 5. It is evident that the mast on the building possesses much lower ability to launch an upward leader as compared to the isolated mast. In the limiting case, as the influence of building vanishes, the ambient potential required for the inception approaches that of an isolated mast.

Application of the present model to the evaluation of the upward leader inception from buildings requires higher computational time. Considering this, for envisaging the applicability of the method, simulations are carried out for a cylindrical stalk of $2 \mathrm{~m}$ radius and $50 \mathrm{~m}$ height. The top of the stalk is flat and the top edge has a circular cross section. The radius of this circular edge is varied and the corresponding leader inception potentials are studied. Owing to the symmetry of the geometry, the inception point is arbitrarily fixed on the middle of the top edge. For simplicity, the corona envelope, (whenever required) is made to have a uniform thickness covering the top edge. For facilitating this, instead of maximum gradient, the average gradient on the top edge is used for the simulation. Simulations are carried out for the corner radii ranging from $5 \mathrm{~cm}$ to $20 \mathrm{~cm}$ in steps of $2.5 \mathrm{~cm}$. The simulations results show that, for the top edge radii greater than or equal to $15 \mathrm{~cm}$, the inception of corona and the leader are coincident. For the edge radius lower than the above, a corona shell appears first, which will be followed by a cylindrical streamer. The computed ambient potential at the inception for the edge radii less than $15 \mathrm{~cm}$ was almost constant and its value is about $2 \mathrm{MV}$. Further, due to a limited extension of the corona envelope, the results for streamer without corona was very close to that with corona envelope. For a further demonstration, the above exercise is repeated for another cylindrical stalk of same radius but $100 \mathrm{~m}$ height. Simulations are carried out with the edge radius as the parameter. For all edge radii less than or equal to $17.5 \mathrm{~cm}$, the corona inception preceded the leader inception and the ambient potential required for the leader inception is found to be around $2.2 \mathrm{MV}$.

In the practical scenario, it becomes necessary to consider the inception from regions of local field intensifications like small metallic projections/protrusions. In this contest, it will be necessary that the method proposed should also work equally well for such geometries. In order to check this, as a first step approximation, small masts of $1 \mathrm{~cm}$ radius are considered. Its length is varied from $30 \mathrm{~cm}$ to $5 \mathrm{~cm}$ in steps of $5 \mathrm{~cm}$ and simulations are carried out. It is found that the proposed model is capable of envisaging inception of upward leaders even though the descending leader position is very close to cause the bridging (i.e., average gradient between leader tip and the point of strike was reaching $500 \mathrm{kV} / \mathrm{m}$ ).

\section{SUMMARY AND CONCLUSIONS}

A more reliable evaluation of the efficacy of the air termination network of lightning protection schemes to taller structures requires a detailed consideration of the inception and propagation of the upward connecting streamers/leaders. For ascertaining the bridging by streamer mechanism, it seems to be adequate to check for the existence of critical average gradient. However, for that with significant upward leader activity, it will be very essential to consider in detail the inception and propagation of upward leaders. This work limits itself to the inception of upward leaders. Owing to the limitations of the earlier works, the present work has made a serious attempt to provide a general macroscopic model for the inception of upward leaders. The proposed model was first scrutinized with the earlier works on switching surge breakdown of long air gaps. It was then shown that for the continuous leader to incept, the length of the streamer in the model proposed should attain a critical value of $2.2 \mathrm{~m}$. Later the model was applied for the study of the upward leader inception from tall masts and conductors. The simulation results were compared reasonably well with the published results for these two geometries. Lastly, for the demonstration, the present model was applied to study the leader inception from some practical lightning protection problems. A few salient cases were also considered to demonstrate the stability of the proposed model.

In conclusions, the present work has successfully proposed a macroscopic model for the evaluation of the upward leader inception. This model is quite general and seems to apply for the upward leader inception from any arbitrary geometry.

For the evaluation of the protection efficacy of protection system to tall structures, the complete bridging needs to be considered. The work is now being continued to address the same.

\section{REFERENCES}

[1] T. Horvath, Computation of Lightning Protection. New York: Wiley, 1991.

[2] R. H. Golde, "Lightning conductor," in Lightning, R. H. Golde, Ed. New York: Academic, 1977, vol. 2.

[3] "Estimating lightning performance of transmission lines-II: Updates to analytical model," IEEE Trans. Power Del., vol. 8, no. 3, pp. 1254-1267, Jul. 1992. 
[4] A. M. Mousa and K. D. Srivastava, "The implications of the electrogeometric model regarding effect of height of structure on the median amplitude of collected lightning strokes," IEEE Trans. Power Del., vol. 4, no. 2, pp. 1450-1460, 1989.

[5] A. J. Eriksson, "An improved electrogeometric model for transmission line shielding analysis," IEEE Trans. Power Del., vol. 2, pp. 871-886, 1987.

[6] L. Dellera and E. Garbagnati, "Lightning stroke simulation by means of the leader progression model, Part I: Description of the model and evaluation of freestanding structures," IEEE Trans. Power Del., vol. 5, pp. 2009-2023, 1990.

[7] F. Rizk, "Modeling of lightning incidence to tall structures, Part I \& II," IEEE Trans. Power Del., vol. 9, no. 1, pp. 162-193, 1994.

[8] — " "Modeling of transmission line exposure to direct lightning strokes," IEEE Trans. Power Del., vol. 5, no. 4, pp. 1983-1997, 1990.

[9] — "A model for switching impulse leader inception and breakdown of long air-gaps," IEEE Trans. Power Del., vol. 4, pp. 596-606, 1989.

[10] U. Kumar and N. T. Joseph, "Analysis of air termination system of the lightning protection scheme for the Indian satellite launch pad," IEE Proc Sci. Meas. Technol., vol. 150, no. 1, pp. 3-10, Jan. 2003.

[11] U. Kumar and G. Ghosh, "On the efficacy of the lightning masts on structures," in Proc. 12th Int. Symp. High Voltage Engineering, Bangalore, India, 2001, 2.25.

[12] F. D. Alessandrov, "Striking distance factors and practical rod installations: A qunatitative study," J. Electrostatics, vol. 59, pp. 25-41, 2003.

[13] R. Brambilla and A. Pigini, "Discharge phenomena in large conductor bundles," in Proc. 4th Int. Conf. Gas Discharges, Swansea, U.K., 1976.

[14] F. D. Alessandrov and J. R. Gumley, "A collection volume method for the placement of air terminals for the protection of structures against lightning," J. Electrostatics, vol. 50, pp. 279-302, 2001.

[15] P. Lalande, A. Bondiou-Clergerie, G. Bacchiega, and I. Gallimberti, "Observations and modeling of lightning leaders," C.R. Phys., vol. 3, pp. 1375-1392, 2002.

[16] A. Bondiou and I. Gallimberti, "Theoretical modeling of the development of the positive spark in long gaps," J. Phys. D: Appl. Phys, vol. 27, pp. 1252-1266, 1994
[17] J. Padhi, "A Novel Method for the Evaluation of Upward Leader Launching Efficacy of Lightning Protection Schemes," Master's, Dept. High Voltage Eng., Indian Institute of Science, 2003.

[18] M. Goldman and R. S. Sigmond, "Corona and insulation," IEEE Trans. Elect. Insul., vol. 17, no. 2, pp. 90-105, Apr. 1982.

Udaya Kumar was born in Udupi, Karnataka, India in 1967. He received the B.E. degree (1989) in electrical engineering from Bangalore University and the M.E. (1991) and Ph.D. (1997) degrees from the Department of High Voltage Engineering, Indian Institute of Science, Bangalore.

He is currently an Assistant Professor in the Department of High Voltage Engineering, Indian Institute of Science, Bangalore. His major areas of interest are modeling of lightning, analysis and design of lightning protection systems, electromagnetic field analysis pertaining to insulation and lightning.

Prasanth Kumar Bokka was born in India in 1979. He received the B.E. degree (2001) in electrical engineering from Andhra University, Visakhapatnam. He is currently pursuing the M.S. degree in the Department of High Voltage Engineering, Indian Institute of Science, Bangalore.

His areas of interest are lightning protection and field computations.

Jagannath Padhi was born in Ganjam, Orissa in 1978. He completed the B.E (Electrical) degree from UCE, Burla, Orissa, and the M.Sc. (Engg.) from the IISc, Bangalore in 2001 and 2003, respectively.

$\mathrm{He}$ is currently working as an Executive Engineer in BHEL, India. His research interests include broad areas of various lightning protection schemes and high voltage engineering. 